\title{
killerFLIP: a novel lytic peptide specifically inducing cancer cell death
}

\author{
B Pennarun ${ }^{1}$, G Gaidos ${ }^{2}$, O Bucur ${ }^{1}$, A Tinari ${ }^{3}$, C Rupasinghe ${ }^{2}$, T Jin ${ }^{1}$, R Dewar ${ }^{1}$, K Song ${ }^{1}$, MT Santos ${ }^{1}$, W Malorni ${ }^{3,4}$, D Mierke ${ }^{2}$ and R \\ Khosravi-Far ${ }^{*, 1,5}$
}

One of the objectives in the development of effective cancer therapy is induction of tumor-selective cell death. Toward this end, we have identified a small peptide that, when introduced into cells via a TAT cell-delivery system, shows a remarkably potent cytoxicity in a variety of cancer cell lines and inhibits tumor growth in vivo, whereas sparing normal cells and tissues. This fusion peptide was named killerFLIP as its sequence was derived from the $C$-terminal domain of $c$-FLIP, an anti-apoptotic protein. Using structure activity analysis, we determined the minimal bioactive core of killerFLIP, namely killerFLIP-E. Structural analysis of cells using electron microscopy demonstrated that killerFLIP-E triggers cell death accompanied by rapid (within minutes) plasma membrane permeabilization. Studies of the structure of the active core of killerFLIP (-E) indicated that it possesses amphiphilic properties and self-assembles into micellar structures in aqueous solution. The biochemical properties of killerFLIP are comparable to those of cationic lytic peptides, which participate in defense against pathogens and have also demonstrated anticancer properties. We show that the pro-cell death effects of killerFLIP are independent of its sequence similarity with c-FLIP $P_{L}$ as killerFLIP-induced cell death was largely apoptosis and necroptosis independent. A killerFLIP-E variant containing a scrambled C-FLIP $P_{L}$ motif indeed induced similar cell death, suggesting the importance of the C-FLIP $P_{L}$ residues but not of their sequence. Thus, we report the discovery of a promising synthetic peptide with novel anticancer activity in vitro and in vivo. Cell Death and Disease (2013) 4, e894; doi:10.1038/cddis.2013.401; published online 31 October 2013

Subject Category: Experimental Medicine

Despite progress in the last decade, with the introduction of targeted therapies, refractory and relapsed cancer remains a leading cause of mortality worldwide. RNA interference, drugs targeting tumor metabolism and cancer immunotherapy hold exciting therapeutic potential but have not yet translated into many clinical successes. ${ }^{1-3}$ For this reason, there is still an urgent need for novel strategies to target cancer cells.

The last few years have seen a renewed interest in therapeutic peptides. Peptides are target selective, easy to synthesize and possess low toxicity profiles. In the past, the use of native peptides has been hampered by their inactivation in serum, sensitivity to enzymatic degradation and rapid clearance from the bloodstream. However, recent technological advances such as the design of stapled peptides, diastereoisomeric peptides, cyclic peptides and the engineering of peptides capable of self-assembling into nanostructures have significantly enhanced their utility by increasing their bioavailability and stability. ${ }^{4-7}$ Some recently discovered peptides act as potent inhibitors and/or induce cell death by various mechanisms in mammalian cells, including cancer cells. $^{7-9}$

Targeting cell death mechanisms in diseases, including cancer, is a promising treatment option. ${ }^{10}$ While trying to identify novel ways to induce cancer cell death, we discovered a 15-mer peptide READFFWSLCTADMS that presented some homology to a peptide isolated by phage display screening and alanine scanning (FWWLXTA). ${ }^{11}$ Our 15-mer peptide corresponds to a stretch of amino-acid residues in the $C$-terminal domain of the long isoform of C-FLICE-like inhibitory protein $\left(\mathrm{c}-\mathrm{FLIP}_{\mathrm{L}}\right){ }^{11} \mathrm{c}-\mathrm{FLIP}_{\mathrm{L}}$ is one of the three spliced variants of $\mathrm{C}-\mathrm{FLIP}$ found at the protein level together with its short isoform (c-FLIP ${ }_{S}$ ) and $c-F_{\text {FIP }}{ }_{\mathrm{R}}{ }^{12}$ Structurally, C-FLIP $P_{L}$ is similar to procaspase 8. It contains two death effector domain domains and a caspase-like domain. This caspase-like domain is replaced by short $C$-terminal sequences in FLIP $_{\mathrm{S}}(26-28 \mathrm{kDa})$ and $\mathrm{c}-\mathrm{FLIP}_{\mathrm{R}}(24 \mathrm{kDa}) .^{12}$

To study potential effects of our C-FLIPL-derived peptide in cancer cells, an $\mathrm{N}$-terminal TAT cell delivery sequence was added to facilitate membrane permeability. Interestingly, our fusion peptide-induced cell death in Jurkat cells in the micromolar range, independently of death receptor stimulation. In this study, we characterized the anticancer properties of this cell death-inducing peptide named killerFLIP in cell-based models. The active motif of this peptide was identified and its mechanism of action determined, namely apoptosis- and necroptosis-independent plasma membrane

\footnotetext{
${ }^{1}$ Department of Pathology, Beth Israel Deaconess Medical Center and Harvard Medical School, Boston, MA, USA; ${ }^{2}$ Department of Chemistry, Dartmouth College, Hanover, NH, USA; ${ }^{3}$ Department of Technology and Health, Istituto Superiore di Sanita', Rome, Italy; ${ }^{4}$ Department of Therapeutic Research and Medicine Evaluation, San Raffaele Institute Sulmona, L'Aquila, Italy and ${ }^{5}$ Biological and Biomedical Sciences Program, Harvard Medical School, Boston, MA, USA

${ }^{*}$ Corresponding author: R Khosravi-Far, Department of Pathology, Beth Israel Deaconess Medical Center and Harvard Medical School, 330 Brookline Avenue, Boston, MA 02215, USA. Tel: +1 617667 8526; Fax: +1 617667 8838; E-mail: rkhosrav@ bidmc.harvard.edu

Keywords: peptide; amphiphilic; death; lysis

Abbreviations: CAP, cationic antimicrobial peptide; c-FLIP, c-FLICE-like inhibitory protein; FADD, Fas-associated death domain; PARP-1, poly (ADP-ribose) polymerase-1; PI, propidium iodide; RIPK1, receptor-interacting protein kinase 1; TRAIL, tumor necrosis factor-related apoptosis inducing ligand

Received 31.5.13; revised 03.9.13; accepted 05.9.13; Edited by A Stephanou
} 
permeabilization of cancer cells. Effects in a HCT116 human tumor xenograft model were confirmed. Herein, we report a novel lytic peptide that specifically induces tumor cell death and offers promising therapeutic potential.

\section{Results}

The c-FLIP $\mathrm{L}_{\mathrm{L}}$-derived fusion peptide induces cell death in cancer cells in vitro. We previously discovered a 15-mer peptide (READFFWSLCTADMS) derived from the $\beta 5$-strand of $C-F_{L I P}{ }^{11}$ that may have effects on cell death pathways (Supplementary Figure 1). When fused with an $N$-terminal TAT delivery sequence (YGRKKRRQRRR), ${ }^{13}$ this novel peptide, under low serum conditions $(0.5-5 \%$ of $F B S)$, induced a concentration-dependent decrease in cell viability in Jurkat cells, as assessed using an ATP-based assay (Figure 1a). At 10-15 $\mu \mathrm{M}$, the fusion peptide but not the TAT-only peptide-induced cell death in $\sim 50 \%$ of Jurkat cells, as measured by Trypan blue exclusion assay using a Bio-Rad TC10 automated cell counter (Figure 1b). As a result of its ability to induce cancer cell death, the fusion peptide was named killerFLIP.

The bioactive core of killerFLIP induces cell death in a range of tumor cells but not in normal cells. To identify the active core of killerFLIP, we generated truncated killerFLIP variants (Figure 2a). All peptides were fused to an $N$-terminal TAT to allow cell delivery. The effects of these variants on cell death were measured in Jurkat cells using propidium iodide (PI) staining. The most effective variants, namely killerFLIP-A, -B and -E, induced $47.6 \%, 62.1 \%$ and $70.5 \%$ cell death, respectively (Figure $2 \mathrm{~b}$, upper right panel). Both killerFLIP-A and -B contain the killerFLIP-E subfragment. It follows that the FFWSLCTA domain is sufficient for the activity of killerFLIP. Loss of the CTA residues resulted in a concomitant loss of activity as cell death was only seen in $15.9 \%$ of Jurkat cells treated with killerFLIP-D. This emphasizes the importance of the $C$-terminal CTA residues. Yet, these CTA residues themselves were not sufficient as killerFLIP-C (CTADMS) did not induce significant cell death (14.6\%). These results were confirmed using a cell viability assay based on relative ATP levels in Jurkat cells (Figure $2 b$, lower right panel). killerFLIP-A, -B and -E induced robust ATP level inhibition compared with control (down to $34 \%, 26 \%$ and $10 \%$, respectively). killerFLIP-D, the killerFLIP-E variant lacking $C$-terminal CTA residues, elicited a significantly weaker decrease in ATP levels compared with killerFLIP-E $(52 \%$ versus $10 \%, P<0.001)$. These results again indicate that killerFLIP-E retains the cell death-inducing core of killerFLIP. Concentration-dependent effects of killerFLIP-E on cell death were confirmed in Jurkat cells using Annexin $\mathrm{V}$ staining, with an $\mathrm{EC}_{50} \sim 10.7 \mu \mathrm{M}\left(\mathrm{EC}_{50}\right.$ : drug concentration that induces Annexin V positivity in $50 \%$ of the cells; Supplementary Figure 2). Western blot analysis in Jurkat cells shows that at early time points (3-6h), killerFLIP-E treatment-induced appearance of both the large active fragment of caspase 3 $(17 / 19 \mathrm{kDa})$ and the large fragment of poly (ADP-ribose) polymerase 1 (PARP-1; 89 kDa; Figure 2c), both hallmarks of apoptosis.

The effects of killerFLIP-E on cell death were further tested in a panel of 14 normal and cancer cell lines (Figure 2d). At $20 \mu \mathrm{M}$, killerFLIP-E-induced potent cell death in leukemia, prostate cancer and colon cancer cells. The effects were particularly dramatic in K562, MV411 and RS411 leukemia cell lines (7.7\%, $10.8 \%$ and $10.4 \%$ Trypan blue-negative cells, respectively). No toxicity was observed in epithelial and endothelial cells (HPDE and HUVEC, respectively), nor in pancreatic cancer and breast cancer cells. Interestingly, no cross-resistance to killerFLIP-E was observed in the tumor necrosis factor-related apoptosisinducing ligand (TRAIL)-resistant PC3-TR cell line compared with its parental PC3 cell line. Overall, killerFLIP-E exhibited moderate to robust effects in 8 of 12 cancer cell lines, and weak effects in two normal cell lines.

killerFLIP-E rapidly induces formation of breaks at the plasma membrane. We next sought to determine the effects on killerFLIP-E at the ultrastructural level. To establish optimal killerFLIP-E treatment duration before electron microscopy analysis, we first examined its effects on Jurkat and K562 cells in a time-dependent manner using Trypan blue exclusion assay. Surprisingly, within 5-15 min
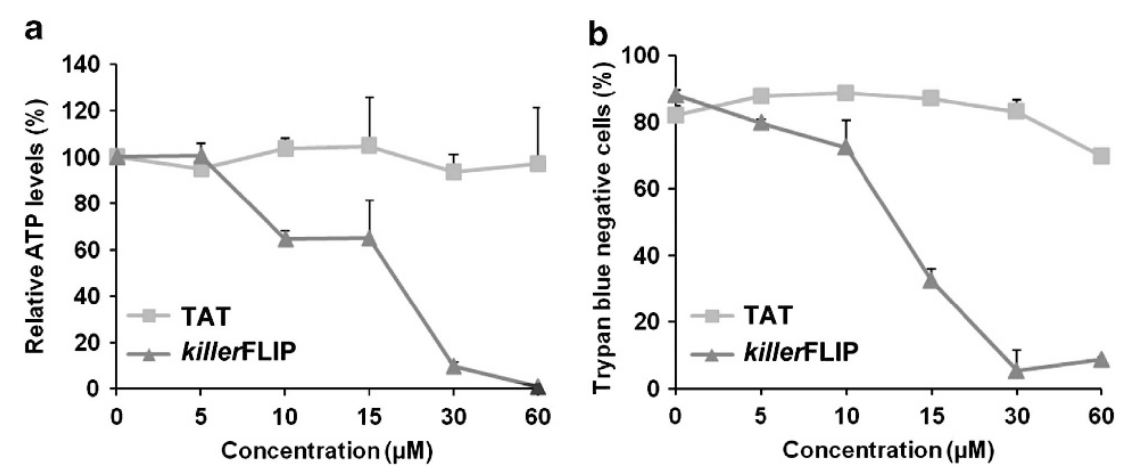

Figure 1 The fusion peptide induces cell death in Jurkat cells. (a) Treatment with fusion peptide decreased cell viability as assessed by relative ATP levels. Jurkat cells were treated overnight with various concentrations of TAT or fusion peptide before ATP levels quantification (expressed as percentage relative to the control). (b) Treatment with fusion peptide-induced cell death as assessed by Trypan blue exclusion assay. Jurkat cells were treated overnight with various concentrations of TAT or fusion peptide before Trypan blue staining and quantification. Data representative of at least two independent experiments performed in triplicate were shown. Values are expressed as mean ( \pm S.D.) 
a

killerFLIP-A: READFFWSLCTADMS killerFLIP-B: killerFLIP-C: killerFLIP-E:
FFWSLCTADMS CTADMS

FFWSL FFWSLCTA killerFLIP-D:

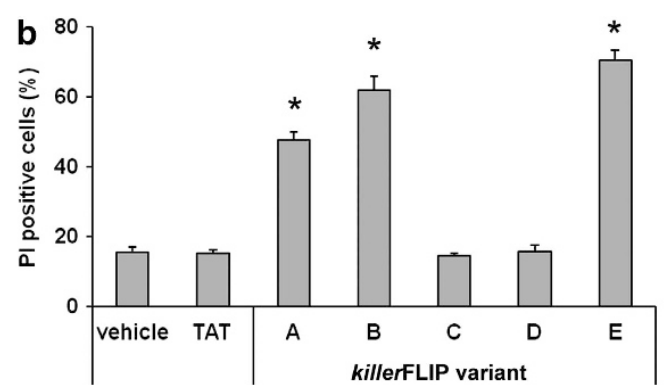

C

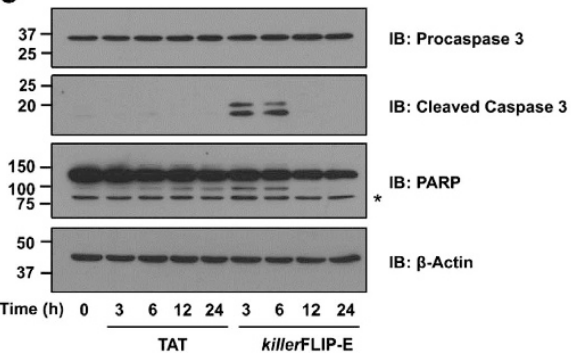

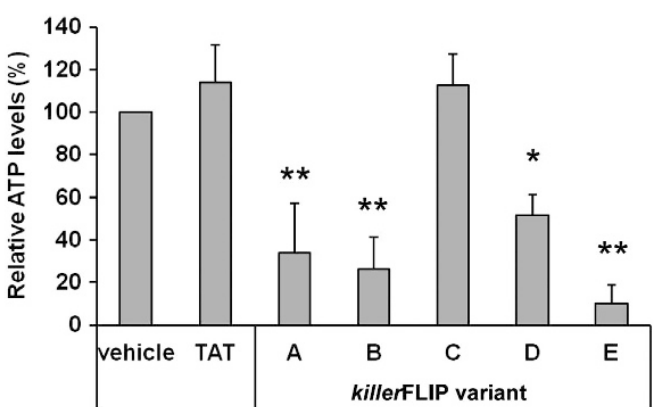

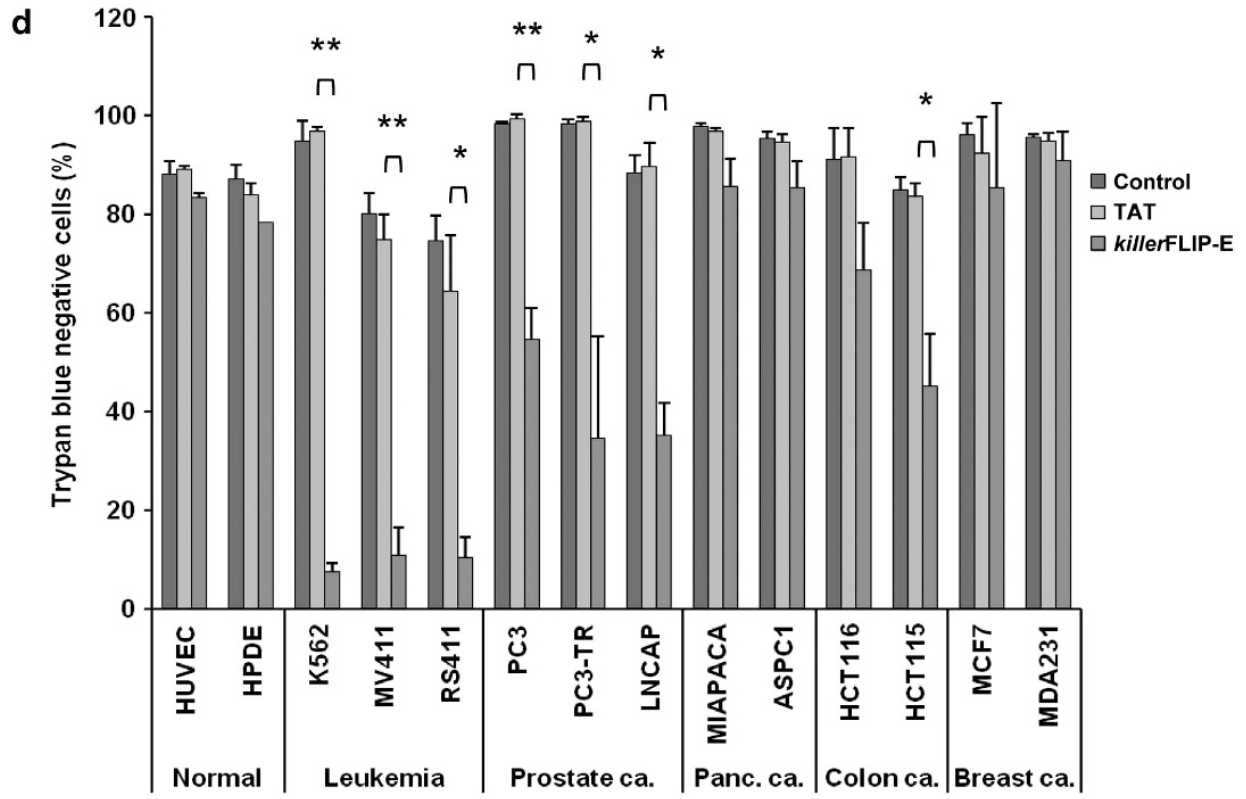

Figure 2 killerFLIP-E is the bioactive motif of the fusion peptide. (a) Amino-acid sequences of the five C-FLIP -derived motifs tested. (b) Jurkat cells were treated with the killerFLIP variants $(15 \mu \mathrm{M})$ for $18 \mathrm{~h}$ before quantification of cell death with PI staining and flow cytometer analysis (top panel). Values are expressed as mean ( \pm S.D.) of three independent experiments. ${ }^{*} P<0.001$ using Student's $t$-test for the comparison between TAT-treated and cells treated with the killerFLIP variants. Jurkat cells were treated with the various peptides $(15 \mu \mathrm{M})$ for $18 \mathrm{~h}$ before ATP quantification (bottom panel). Values are expressed as mean ( \pm S.D.) of four independent experiments. ${ }^{*} P<0.05$ and ${ }^{* *} P<0.005$ using Student's $t$-test for the comparison between TAT-treated and cells treated with the killerFLIP variants. (c) Western blot analysis showing time-dependent effects of TAT and killerFLIP-E (both $15 \mu \mathrm{M}$ ) on caspase 3 and PARP-1 cleavage in Jurkat cells; *Indicates an aspecific band. Data are representative of three independent experiments. (d) Effects of $18 \mathrm{~h}$ killerFLIP-E treatment $(20 \mu \mathrm{M})$ on cell death as assessed by Trypan blue exclusion assay in a panel of normal and cancer cell lines; ${ }^{*} P<0.01$ and ${ }^{* \star} P<0.001$ (Student's $t$-test). Data represent mean ( \pm S.D.) of at least two independent experiments

killerFLIP-E induced a drastic drop in the percentage of Trypan blue-negative Jurkat cells (Figure 3a). Following 15 -min treatment with $20 \mu \mathrm{M}$ killerFLIP-E, only $21.8 \%$ of Jurkat cells remained Trypan blue-negative compared with $75.2 \%$ of TAT-treated cells. Similar effects were seen in K562 cells $(22.4 \%$ versus $83 \%$ of TAT-treated cells; Figure $3 b)$. These results indicate that killerFLIP-E causes rapid loss in plasma membrane integrity in cancer cells.
As killerFLIP-E-induced cell death within $15 \mathrm{~min}$, Jurkat cells were treated with TAT and killerFLIP-E for $5 \mathrm{~min}$, and the early effects on subcellular structures examined using electron microscopy. In cells treated with TAT, typical T-cell ultrastructural features, for example, a low nuclear/cytoplasmic ratio, were observed (Figure 3c, left panel). However, dramatic alterations were seen in Jurkat cells treated with killerFLIP-E (Figure 3c, right panel). Single membrane 

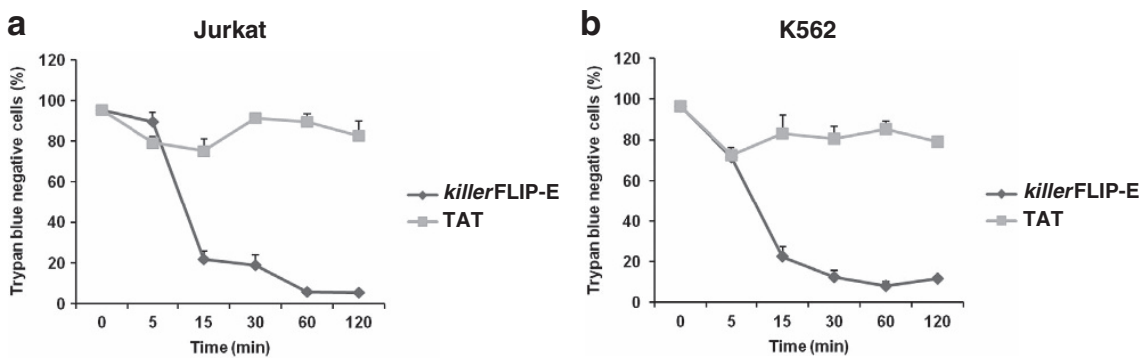

C

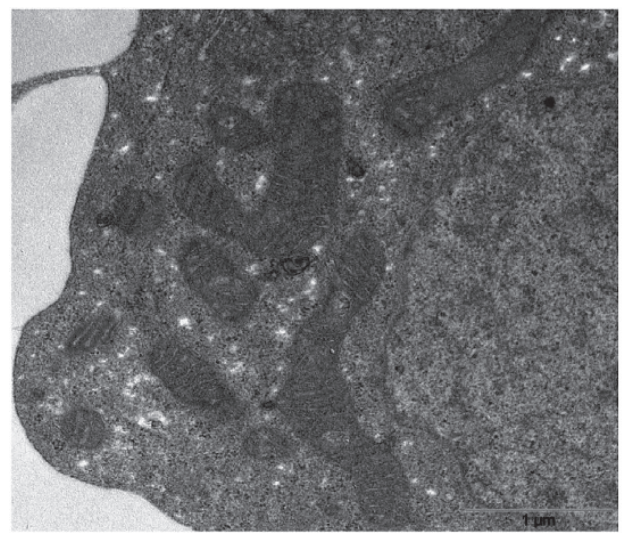

TAT

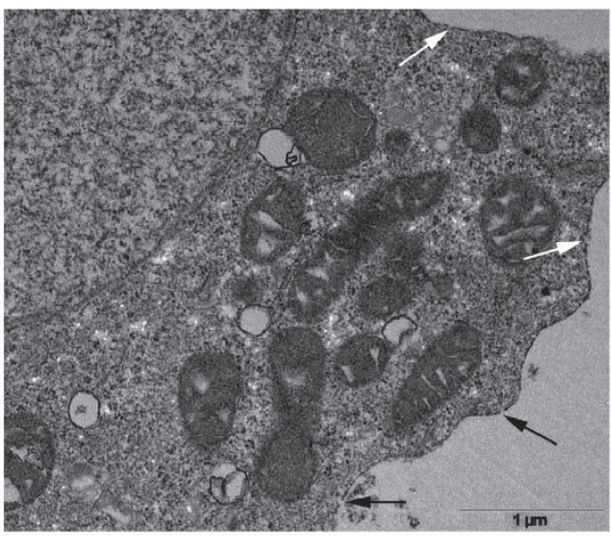

killerFLIP-E

Figure 3 killerFLIP-E rapidly disrupts plasma membrane integrity and induces ultrastructural alterations in cancer cells. (a) Jurkat and (b) K562 cells were treated with $20 \mu \mathrm{M}$ TAT or killerFLIP-E for the indicated times before Trypan blue exclusion assay. Values expressed as mean ( \pm S.D.). (c) EM micrographs showing differences in Jurkat cell morphology upon exposure to $20 \mu \mathrm{M}$ TAT (left) and killerFLIP-E (right) for $5 \mathrm{~min}$

(therefore non-autophagic) vesicules were observed, some of them partly associated with the mitochondria. Mitochondria exhibited increased electron density with altered cristae and rarefied matrix. Interestingly, breaks were seen at the plasma membrane (black and white arrows) while the nucleus was intact (upper left corner). At later time-points (30 min), Jurkat cells treated with killerFLIP-E displayed features indicative of late necrosis (data not shown). We also examined the effects of killerFLIP-E at the ultrastructural level in MCF7 breast cancer cells, which were mostly resistant to killerFLIP-E (Figure 2d and Supplementary Figure 3). At $30 \mathrm{~min}, \mathrm{MCF} 7$ cells treated with killerFLIP-E still exhibited typical ultrastructural features. Importantly, no plasma membrane breaks were seen. This indicates that membrane breaks are only induced in cell lines sensitive to killerFLIP-Einduced cell death.

killerFLIP-E corresponds to a novel cancer cell lytic peptide. Cationic antimicrobial peptides (CAPs) are known to permeabilize cell membranes, including cancer cell membranes. ${ }^{14,15}$ CAPs have an amphiphilic structure because of positively charged residues and a large portion of hydrophobic residues. The eight arginine $(R)$ and lysine (K) residues in the TAT domain confer killerFLIP-E a +8 net charge, in line with the net positive charge of CAPs ranging from +2 to $+9^{16}$ (Figure $4 \mathrm{a}$ ). In addition to the proline $(P)$ residue at the $C$-terminal of the TAT domain, the $\mathrm{C}-\mathrm{FLIP}_{\mathrm{L}}$ derived domain of killerFLIP-E is mostly hydrophobic owing to the presence of phenylalanine $(F)$, tryptophan $(W)$, leucine
(L) and alanine (A) residues. killerFLIP-E thus possesses the characteristics of an amphiphilic peptide, and might therefore form micelles in solution. Using DLS, we confirm that killerFLIP-E molecules self-assemble in aqueous solution into micelles, spherical and/or cylindrical structures of a relatively homogeneous diameter $(804 \pm 236 \mathrm{nM})$, whereas the control peptide TAT does not (Figure 4b).

To investigate the structure-function relationship of killerFLIP-E, we generated a variant containing a scrambled C-FLIPL-derived fragment (FALFCTSW). We then tested the effects of this scrambled killerFLIP-E on cell viability using an ATP-based assay in Jurkat cells. Treatment with killerFLIP-E and scrambled killerFLIP-E induced similar decreases in relative ATP levels compared with vehicle-treated cells (26.6\% and $12.7 \%$, respectively; Figure $4 \mathrm{c})$. This strongly suggests that the biochemical properties of the amino-acid residues in killerFLIP-E are more critical than their order in the $C$-terminal killerFLIP-E domain.

killerFLIP-E induces cell death in a caspase-independent and receptor-interacting protein kinase 1 (RIPK1)independent manner. $C-F_{L} P_{L}$ is a critical protein influencing death receptor-mediated apoptosis. ${ }^{17} \mathrm{C}-\mathrm{FLIP}_{\mathrm{L}}$ modulates caspase 8 activation within membrane complexes called death-inducing signaling complexes comprised of death receptors and adapter proteins such as Fas-associated death domain (FADD). killerFLIP-E induced some caspase 3 and PARP-1 cleavage at 3 and $6 \mathrm{~h}$ (Figure 2c), indicating apoptosis activation. Day et al. ${ }^{18}$ suggested that $\mathrm{C}-\mathrm{FLIP} \mathrm{P}_{\mathrm{L}}$ 
TAT C-FLIP derived motif
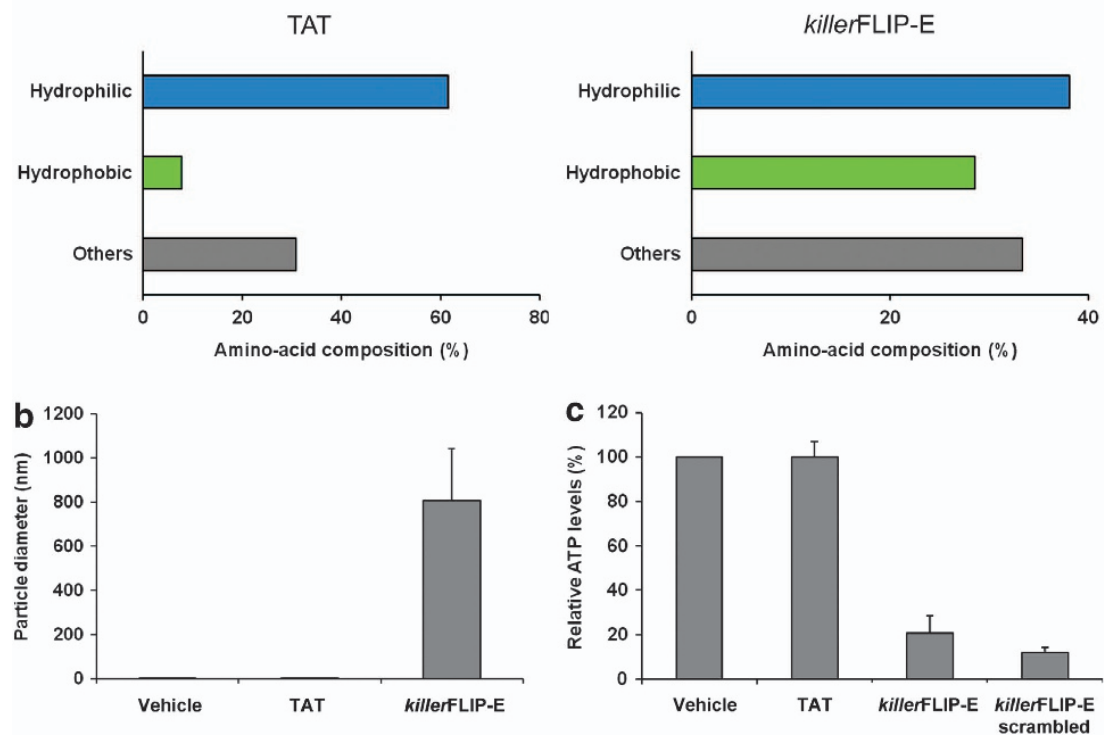

Figure 4 The hydrophobic profile of killerFLIP-E is more important than its sequence for the effects on cell death. (a) The hydrophobic profiles of TAT and killerFLIP-E were analyzed. (b) Measurement of TAT and killerFLIP-E particule size using DLS. The mean ( \pm S.D.) is shown ( $n \geq 7)$. (c) killerFLIP-E and scrambled killerFLIP-E had similar effects on cell death in Jurkat cells as assessed by relative ATP levels. Cells were treated for $18 \mathrm{~h}$ with TAT, killerFLIP-E or a scrambled killerFLIP-E before quantification of the relative ATP levels

might form an apoptotic inhibitory complex (AIC) with DR5, FADD and caspase-8 in cancer cells. C-FLIP $\mathrm{L}$ knockdown induced ligand-independent apoptosis in a DR5-, FADD- and caspase 8-dependent manner. We reasoned that because of its analogy with $\mathrm{C}-\mathrm{FLIP}_{\mathrm{L}}$, killerFLIP-E might disrupt recruitment of $C-F L I P L$ to these AICs and thereby activate ligandindependent apoptosis. We utilized FADD- and caspase 8deficient Jurkat cell models to test the importance of some key proteins found in the AIC for killerFLIP-E sensitivity. killerFLIP-E treatment induced comparable decreases in cell viability as assessed by ATP levels in parental (clone A3), FADD-deficient and caspase 8-deficient Jurkat cells $\left(\mathrm{IC}_{50} \sim 26,20\right.$ and $28 \mu \mathrm{M}$, respectively) $\left(\mathrm{IC}_{50}\right.$ : the drug concentration causing a $50 \%$ inhibition in relative ATP levels; Figure 5a). This suggests that FADD and caspase 8 do not have a role in killerFLIP-E-induced cell death. To further analyze the role of caspases, we pre-treated Jurkat cells with the pancaspase inhibitor zVAD-fmk and tested the effects on killerFLIP-E sensitivity. Cells were also treated with HGSETR2, an agonistic anti-TRAIL-R2 antibody used as a positive control for apoptotic cell death. Treatment with $10 \mu \mathrm{M}$ TAT did not induce cell death as measured by Trypan blue exclusion (Figure 5b). Treatment with $7.5 \mu \mathrm{M}$ killerFLIP$E$ induced a decrease in the percentage of cells excluding Trypan blue compared with TAT, from $78 \%$ to $15 \%$. zVAD-fmk pre-treatment $(20 \mu \mathrm{M})$ significantly impaired HGS-ETR2-induced cell death from $11 \%$ to $70 \%$ Trypan blue-negative cells $(P<0.001)$. Pre-treating Jurkat cells with $20 \mu \mathrm{M}$ zVAD-fmk is therefore sufficient to prevent caspasemediated cell death. However, pre-treatment with $20 \mu \mathrm{M}$ (and $50 \mu \mathrm{M}$ ) zVAD-fmk did not inhibit the effects of killerFLIP-E
(11\% Trypan blue-negative cells in both treatment groups). These results show that killerFLIP-E-induced cell death is largely caspase independent.

A caspase-independent pathway termed regulated necrosis (or necroptosis) can be activated by death receptors in conditions under which apoptosis are blocked. ${ }^{19}$ Necroptosis activation involves formation of a complex called the necrosome that consists of RIPK1 and RIPK3. Although the detection of some caspase 3 cleavage suggested otherwise (Figure 2c), it could not be excluded that killerFLIP-E might activate necroptosis. To test this hypothesis, we assessed the effects of killerFLIP-E in a RIPK1-deficient Jurkat model. killerFLIP-E induced similar decreases in relative ATP levels in both parental (clone SVT35) and RIPK1-deficient Jurkat cells $\left(\mathrm{IC}_{50} \sim 9 \mu \mathrm{M}\right.$; Figure $\left.5 \mathrm{c}\right)$. This shows that the effects of killerFLIP-E on cell viability are independent of necrosome formation.

killerFLIP is well tolerated in vivo and inhibits tumor growth. We next tested the effects of killerFLIP in vivo in a HCT116 human colon tumor xenograft mouse model. Animals were injected daily with TAT $(1.3$ and $5.3 \mathrm{mg} / \mathrm{kg}$ ) or killerFLIP (2.0 and $8.0 \mathrm{mg} / \mathrm{kg}$ ) for 18 days. No significant variation in body weight was detected in mice treated with TAT or killerFLIP (Figure 6a). Furthermore, no signs of toxicity were observed in H\&E-stained liver sections of animals killed after 18 days of treatment with vehicle, TAT or killerFLIP (Figure 6b). Similar findings were made in H\&E-stained brain, heart, lung and spleen tissue sections (data not shown). Thus, killerFLIP treatment was generally well tolerated in mice treated for 18 days. 
a
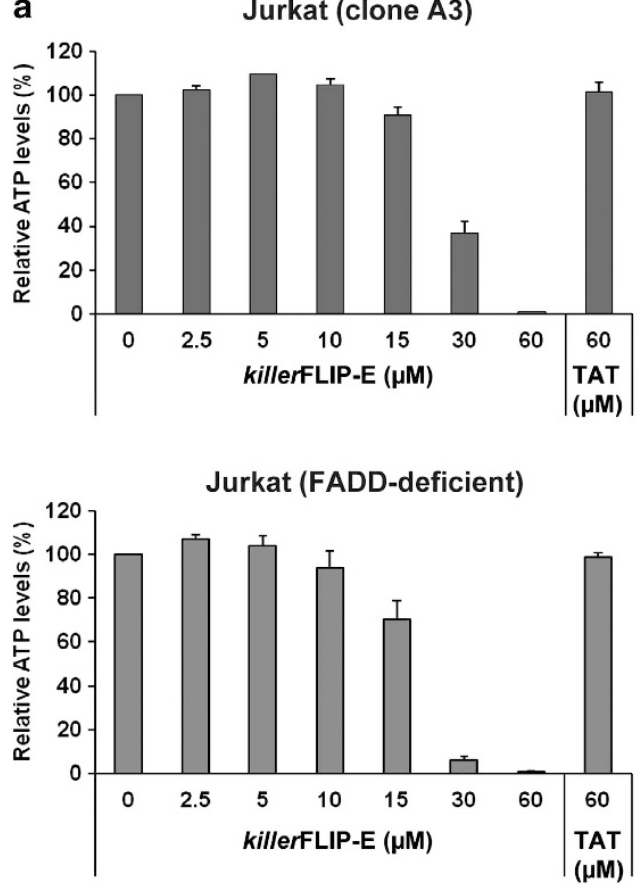

b

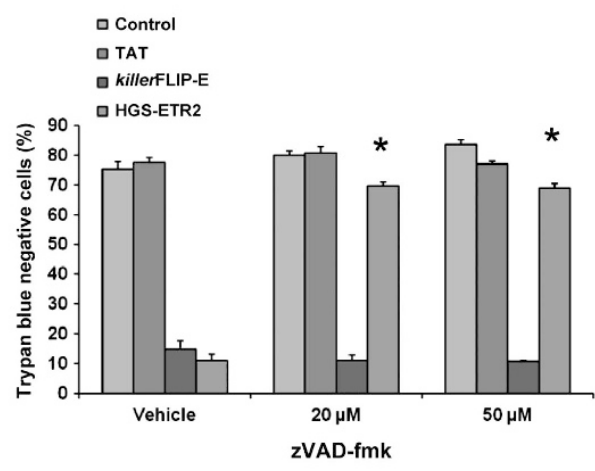

Jurkat (caspase 8-deficient)
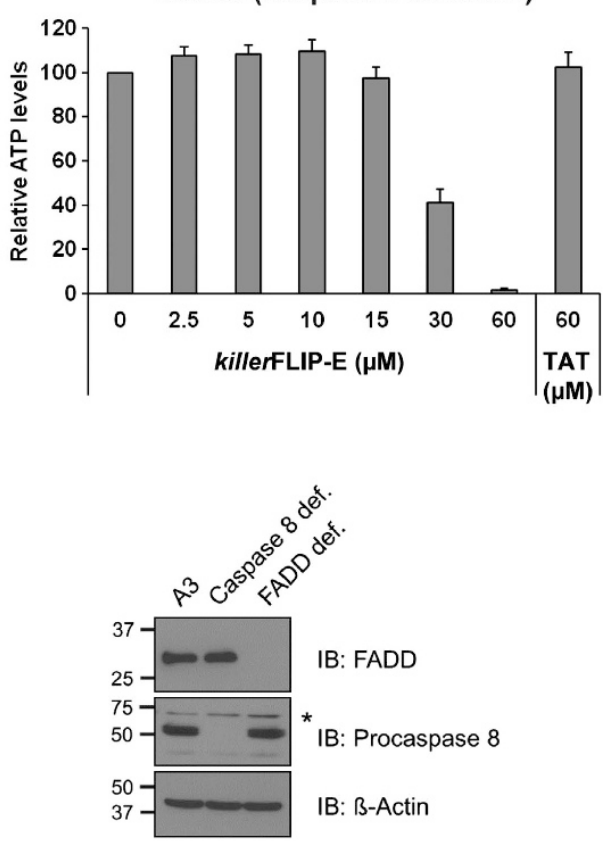

C

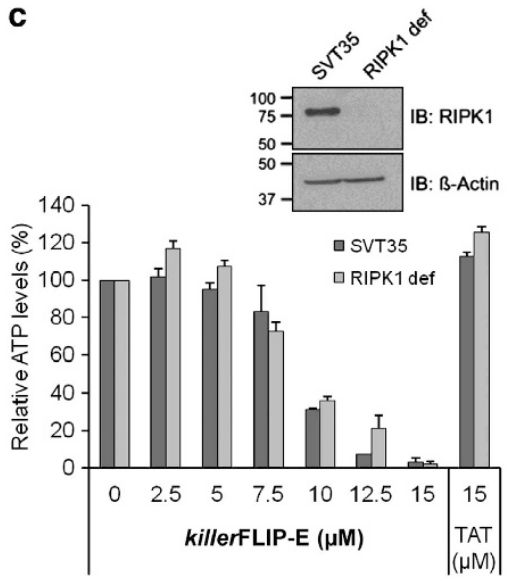

Figure 5 killerFLIP-E induces cell death independently of apoptosis and necroptosis. (a) Effects of TAT or killerFLIP-E in parental Jurkat cells, FADD-deficient Jurkat cells and caspase 8-deficient Jurkat cells as assessed by ATP levels. The results are expressed as the percentage of ATP signal in treated cells relative to vehicle-treated controls. Lack of caspase 8 and FADD protein expression in caspase 8-deficient (caspase 8 def.) and FADD-deficient (FADD def.) Jurkat cells was confirmed by western blot analysis; " Indicates an aspecific band. (b) Effects of pre-treatment with various concentrations of the pancaspase inhibitor zVAD-fmk on cell death induced by vehicle, TAT $(10 \mu \mathrm{M})$, killerFLIP-E $(7.5 \mu \mathrm{M})$ or HGS-ETR2 $(1 \mu \mathrm{g} / \mathrm{ml})$ in Jurkat cells, as assessed by Trypan blue staining and quantification. Cells were preincubated with zVAD-fmk for 30 min before $17 \mathrm{~h}$ treatment with TAT, killerFLIP-E or HGS-ETR2. Data representative of at least three independent experiments. ${ }^{\star} P<0.001$ using Student's $t$-test for the comparison between vehicle-treated groups and cells pre-incubated with ZVAD-fmk. (c) Parental Jurkat cells (SVT35) and RIPK1 $-/$ - cells were treated with various concentrations of killerFLIP-E or $15 \mu \mathrm{M}$ TAT before relative ATP level quantification. Data representative of two independent experiments are shown as mean ( \pm S.D.). Lack of RIPK1 protein expression in RIPK1 deficient (RIPK1 def.) Jurkat cells was confirmed by western blot analysis

More significantly, tumor growth was strongly inhibited in mice treated with killerFLIP compared with TAT control peptide (Figure 7a). Following formalin fixation and paraffin embedding of the tumors from different treatment groups, a TUNEL assay was performed. Tumor sections from mice treated with killerFLIP showed increased positive staining for TUNEL compared with vehicle and TAT-treated groups (Figure 7b). Overall, our results show that killerFLIP can prevent human cancer cell growth in vivo.

\section{Discussion}

Here, we show that a fusion peptide comprised of a TAT cell delivery sequence and a 15-mer domain corresponding to the $\beta 5$-strand of $\mathrm{c}$-FLIP ${ }_{\mathrm{L}}^{11}$ induces cell death in cancer cells in vitro and prevents tumor growth as single agent in an established HCT116 human colon tumor xenograft model. Using truncated variants of this killerFLIP fusion peptide, we found that the E fragment (FFWSCLTA) constitutes the active 


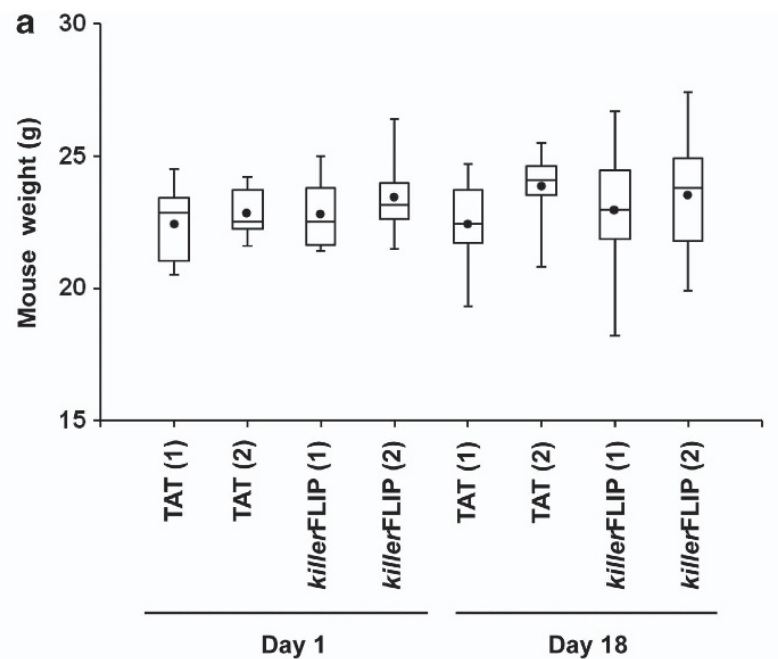

b

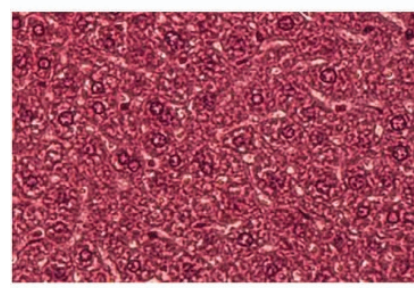

Mock

H\&E staining (liver)

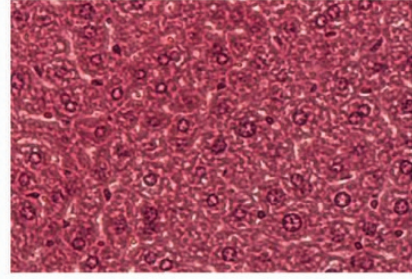

TAT

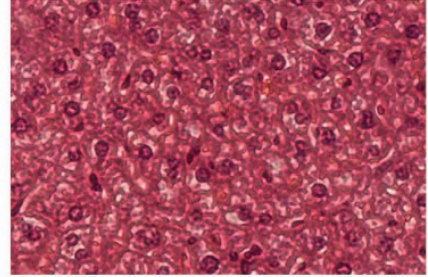

killerFLIP

Figure 6 killerFLIP was well tolerated in mice. (a) Box plots showing weights of experimental animals before and after treatment with the indicated peptides. Four groups of eight mice each were weighed on the first and last days (day 18) of treatment. Black dots represent mean for each treatment group. (b) Representative liver sections from the indicated experimental groups of mice stained with H\&E

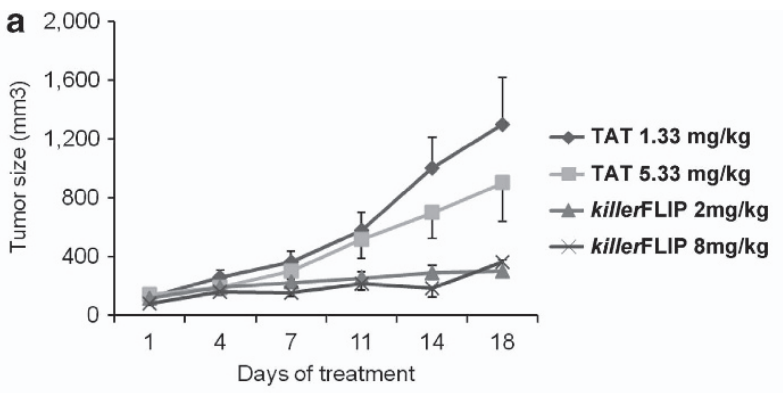

b

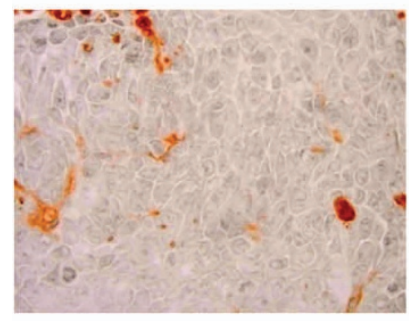

Mock

TUNEL staining (tumor)

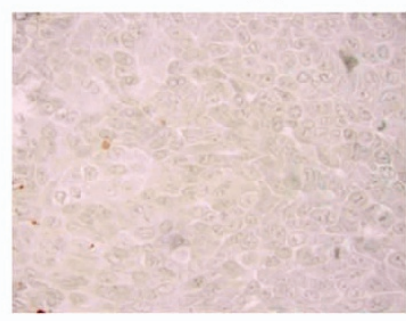

TAT

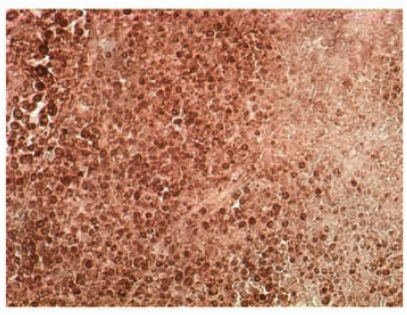

killerFLIP

Figure 7 Growth of established HCT116 xenograft is significantly impaired in response to killerFLIP. (a) Mice subcutaneously implanted with HCT116 xenograft were intraperitoneally administered once per day with the indicated doses of TAT or killerFLIP for 18 days. Tumor size was measured as described in Material and Methods section. (b) Treatment with killerFLIP-induced cell death in xenograft tumors. Histological analysis of biopsy samples from the mice treated with vehicle, TAT or killerFLIP. Tumor sections from untreated or mice injected with TAT or killerFLIP for 18 days were stained using TUNEL assay 
motif of the full-length $\mathrm{C}-\mathrm{FLIP}_{\mathrm{L}}$-derived peptide. Investigating early events of killerFLIP-E-induced cell death yielded critical insights into its effects on cancer cells. We established using Trypan blue exclusion assay and electron microscopy that killerFLIP-E induces rapid (5-15 min) membrane permeabilization in some cancer cells. killerFLIP-E has an amphiphilic structure owing to its cationic TAT $N$-terminal domain and its

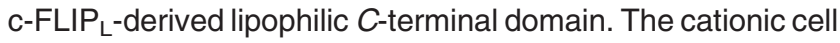
entry peptide TAT alone did not induce cell death or cause breaks in the plasma membrane. An analog of killerFLIP-E containing a scrambled $\mathrm{C}$-FLIP $\mathrm{L}_{\mathrm{L}}$-derived domain triggered similar levels of cell death as killerFLIP-E itself. Thus, our data indicate that the effects of killerFLIP-E do not depend on the sequence of its C-FLIP-derived residues but on their hydrophobic properties.

The amphiphilic structure of killerFLIP-E is analogous to that of CAPs. CAPs are components of the innate immune response that have been identified in every kingdom tested including bacteria, fungi, plants and animals. ${ }^{15}$ The major difference is that the biological activity of the CAPs is sequence and structure dependent. It is only upon folding into an alpha-helix, which readily occurs upon interaction with a membrane, that CAPs present an amphipathic nature, with one face of the helix very hydrophobic, the other containing charged cationic residues. Some CAPs, including hybrid and synthetic CAPs, have been shown to exhibit anticancer properties. Interestingly, the multidrug resistance mechanisms found in cancer cells do not seem to affect the response to these CAPs. We confirmed this finding in the PC3/PC3-TR prostate cancer cell model in regard to TRAIL resistance. Two main models of action of CAPs have been proposed, namely the 'barrel-stave' and the 'carpet' model. ${ }^{14,15}$ These two models describe an initial electrostatic interaction of the cationic peptides with the negatively charged cancer cell membrane. This event is followed by the insertion of the amphiphilic peptide into the plasma membrane through hydrophobic and van der Waals interactions, which induce pore formation. ${ }^{16}$ In the carpet model, CAPs first align parallel to the membrane. In the barrel-stave model, CAPs directly aggregates in the lipid bilayer. In both models, transmembrane pores are formed once a critical peptide concentration is reached. A 'detergent-like' model has also been proposed whereby CAPs intercalate between the phospholipid head groups and induce fragmentation of the lipid bilayer into micelle-like structures. ${ }^{14}$ This model would be consistent with our data showing that killerFLIP-E itself can form micelles in aqueous solution. The disruption of the electrochemical gradient by CAPs results in water and ion flow across the plasma membrane, which cause swelling and bursting of the cell. ${ }^{15}$ Electron microscopy images indeed showed that killerFLIP-E rapidly induced formation of breaks in the plasma membrane of killerFLIP-E-sensitive Jurkat cells but not in resistant MCF7 cells.

killerFLIP-E triggers cell death in a wide panel of cancer cell lines including leukemia, prostate and colon cancer but not in normal epithelial and endothelial cells. killerFLIP appeared well tolerated in mice. The outer leaflet of the plasma membrane of normal eukaryote cells consists predominantly of zwitterionic phospholipids, whereas the outer leaflet of cancer cell membranes is enriched in negatively charged phospholipids. This difference in charge between normal and cancer cell plasma membranes is critical for the selectivity of CAPs. ${ }^{20,21}$ The increased phosphatidylserine content (3-9\%) of the outer leaflet of the plasma membrane in cancer cells notably contributes to this negative charge. ${ }^{16}$ Heparan sulfate and chondroitin sulfate are two major classes of glycosaminoglycan side-chains of proteoglycans. ${ }^{22}$ The sulfate groups provide a highly negative charge to these proteoglycans. ${ }^{23}$ Differences in proteoglycan expression pattern have been observed between normal and cancer cells. ${ }^{24-26}$ Interestingly, both lytic peptides and TAT are known to bind heparan sulfate. $^{27-29}$ However, it is not clear yet whether binding facilitates cellular entry or whether it sequesters the peptides away from the lipid bilayer.

Owing to activation of particular glycotransferases, the surface of cancer cells is also enriched in acidic components such as O-glycosylated mucines with increased terminal sialylation, which contributes to their negative charge. ${ }^{15}$ Although $\alpha 2,3$-sialic acids have been found at the cell surface of MCF7 breast cancer cells, ${ }^{30,31}$ MCF7 cells were resistant to killerFLIP-E. Selective binding and activity of the CAPs might not be explained solely by the higher negative charge of the outer leaflet of the lipid bilayer in cancer cells as some CAPs can permeabilize both negatively charged membranes and zwitterionic membranes. ${ }^{5}$ The number of microvilli has also been suggested to have a role in sensitivity to CAPs. Cancer cells are characterized by a large number of microvilli, which increases their surface area and might increase the amount of peptide at the cell surface. This would in turn allow reaching the critical threshold necessary to initiate permeabilization. ${ }^{5}$ We observed a higher number of microvilli in MCF7 cells compared with Jurkat cells, suggesting that microvilli might not have a critical role in killerFLIP-E sensitivity.

It is remarkable that not every type of cancer cell tested in this study was sensitive to killerFLIP-E. Pancreatic and breast cancer cells in particular were resistant to killerFLIP-E. We cannot rule out the possibility that cancer cell growth features such as anchorage dependence, ${ }^{32}$ formation of tight endosomal intercellular adhesions ${ }^{33}$ or scattered monolayers, could represent key variables in killerFLIP-E susceptibility. Interestingly, leukemia cell lines, which are typically non-adherent, represented the most sensitive group of cells in our panel. It will be critical to determine the nature of the plasma membrane molecules underlying killerFLIP-E sensitivity in leukemia, prostate and colon cancer cells.

Although the plasma membrane was the primary compartment affected by killerFLIP-E, dramatic changes were also observed at the mitochondrial level. The cell entry peptide TAT is known to target therapeutic cargo to the mitochondrial membrane. ${ }^{34}$ This suggests a high affinity of killerFLIP-E for lipid bilayers, including the mitochondrial membrane. Certain death stimuli activate the intrinsic pathway of apoptosis by disrupting the outer mitochondrial membrane, resulting in release of cytochrome $c$ and other pro-apoptotic proteins. Cytosolic cytochrome c binds apoptotic-protease-activating factor 1 within a complex called the apoptosome to activate caspase-9, in turn leading to activation of effector caspases such as caspase 3 . Caspase 3 cleavage by killerFLIP-E might therefore be a consequence of outer mitochondrial membrane permeabilization and subsequent activation of the intrinsic 
pathway of apoptosis. ${ }^{35}$ Importantly, our data show that killerFLIP-induced cell death is mostly due to direct plasma membrane permeabilization, not activation of apoptosis or necroptosis. Developments in the field of biochemical engineering such as the design of diastereoisomeric, cyclic or self-assembling peptides have fueled a renewed interest in peptides as therapeutics. ${ }^{4-6,9}$ A phase I dose escalation study with a lytic peptide (LTX-315) was recently completed in patients with transdermally accessible tumors (Clinicaltrials.gov identifier: NCT01058616) and several antimicrobial peptides recently demonstrated efficacy in phase II/III clinical trials. ${ }^{36}$ In conclusion, synthetic lytic peptides such as killerFLIP-E that specifically target the plasma membrane of cancer cells constitute an exciting class of new anticancer drugs. Our data support further evaluation of the therapeutic potential of killerFLIP-E in cancer.

\section{Materials and Methods}

Reagents. TAT (YGRKKRRQRRR), killerFLIP (YGRKKRRQRRRREADFF WSLCTADMS) and its variants were made at Dr. Mierke's lab at Dartmouth, $\mathrm{NH}$, USA or purchased from Anaspec (Fremont, CA, USA) and Biomatik (Cambridge, ON, Canada). Anti-procaspase 3, active caspase-3, PARP and caspase 8 antibodies were obtained from Cell Signaling Technology (Danvers, MA, USA). Anti-RIPK1 antibody was purchased from BD Transduction Laboratories (San Jose, CA, USA). Anti- $\beta$-actin was from Sigma-Aldrich (St. Louis, MO, USA). PI was purchased from Roche (Indianapolis, IN, USA). The pan-caspase inhibitor Benzyloxycarbonyl-Val-Ala-Asp (OMe)-fluoromethylketone (zVAD-fmk) was from R\&D Systems (Minneapolis, MN, USA). The HGS-ETR2 antibody was a kind gift from Human Genome Sciences (Rockland, MD, USA).

Cell lines. Cells were obtained from the American Type Culture Collection (Manassas, VA, USA) and maintained at low passage number; lines used were HPDE, K562, MV411, RS411, LNCAP, MIAPACA, ASPC1, HCT116, HCT115, MCF7 and MDA-MB-231. Human Umbilical Vein Endothelial Cells (HUVEC) were obtained from Dr. Jack Lawler (Boston, MA, USA). PC3 and PC3-TR cells were a gift from Dr. Aria Olumi (Boston, MA, USA). Parental Jurkat cells (A3), FADD-deficient and caspase 8-deficient Jurkat cells were provided by Dr. John Blenis (Boston, MA, USA). Parental (SVT35) and RIPK1-deficient Jurkat cells were a gift from Dr. Brian Seed (Boston, MA, USA). K562, Jurkat, MV411, RS411, PC3, PC3-TR and LNCAP cells were grown in RPMI supplemented with 10\% FBS and $1.5 \mathrm{mmol} / \mathrm{l}$ L-glutamine. MIAPACA, ASPC1, HCT116, HCT115, MCF7 and MDA231 cells were cultured in DMEM supplemented with 10\% FBS and $1.5 \mathrm{mmol} / \mathrm{l}$ L-glutamine. HCT116 and HCT115 cells were cultured in McCoy's Medium supplemented with $10 \% \mathrm{FBS}$ and $1.5 \mathrm{mmol} / \mathrm{l}$ L-glutamine. HUVEC were grown in M131 medium (Invitrogen, San Diego, CA, USA) supplemented with 5\% FBS and $1.5 \mathrm{mmol} / \mathrm{l}$ L-glutamine. HUVEC cells were grown in dishes and plates pre-coated for $30 \mathrm{~min}$ with $1 \mathrm{X}$ Attachment factor from Cascade Biologics (Invitrogen). HPDE cells were grown in DMEM supplemented with $1 \mathrm{X}$ Keratinocyte-SFM and $1 \mathrm{X}$ microvascular growth supplement (Invitrogen). All growth media included $100 \mathrm{units} / \mathrm{ml}$ penicillin and $100 \mu \mathrm{g} / \mathrm{ml}$ streptomycin. All cell lines were maintained at $37^{\circ} \mathrm{C}$ in a humidified atmosphere with $5 \% \mathrm{CO}_{2}$ All in vitro treatments in this study were performed in medium containing $0.5 \%$ FBS

Sub-G1 analysis. Cells $\left(4 \times 10^{5} / \mathrm{ml}\right)$ were seeded in 12 -well plates before treatment with TAT or the indicated fusion peptides. Cells were then collected and washed once in PBS. The cells were resuspended in $400 \mu \mathrm{l} \mathrm{PI}$ solution $(50 \mu \mathrm{g} / \mathrm{ml}$, $0.1 \%$ sodium citrate and $0.1 \%$ Triton $\mathrm{X}-100$ ) before quantification using a FACSCalibur flow cytometer and CellQuest software (BD Bioscience, San Jose, CA, USA).

Western blot analysis. Cell extracts were lysed for $30 \mathrm{~min}$ on ice in radioimmunoprecipitation assay buffer containing $50 \mathrm{mM} \mathrm{Tris-HCl}(\mathrm{pH} 7.4)$, $150 \mathrm{mM} \mathrm{NaCl}, 1 \% \mathrm{NP}-40,0.5 \%$ sodium deoxycholate, $0.1 \%$ SDS (Boston Bioproducts, Ashland, MA, USA) supplemented with $5 \mathrm{mM}$ EDTA, proteCEASE (G-Biosciences, St. Louis, MO, USA), Halt phosphate inhibitor cocktail (Pierce, Rockford, IL, USA) and $1 \mathrm{mM}$ phenylmethylsulfonyl fluoride. Lysates were cleared at $13200 \mathrm{~g}$ for $10 \mathrm{~min}$ at $4{ }^{\circ} \mathrm{C}$. Proteins were quantified with the BCA protein assay (Pierce) and diluted to a concentration of $1 \mu \mathrm{g} / \mu \mathrm{l}$ in $1 \times$ Laemmli's SDS-sample buffer containing $62.5 \mathrm{mM}$ Tris- $\mathrm{HCl}(\mathrm{pH} 6.8), 2 \%$ SDS, $10 \%$ glycerol, $2 \% \beta$-mercaptoethanol and $0.005 \%$ bromophenol (Boston Bioproducts). Samples were heated to $95^{\circ} \mathrm{C}$ for $3-5 \mathrm{~min}$. Proteins (20-50 $\mu \mathrm{g}$ per sample) were resolved by SDS-PAGE and transferred to polyvinylidene difluoride membranes (Millipore, Bedford, MA, USA). Nonspecific binding sites were blocked with $5 \%$ nonfat dry milk (Bio-Rad, Hercules, CA, USA) in TBS with $0.05 \%$ Tween-20 (Fisher Scientific, Billerica, MA, USA) for $1 \mathrm{~h}$ at room temperature. After blocking, the membranes were incubated with specific antibodies overnight at $4{ }^{\circ} \mathrm{C}$. The horseradish peroxidase-labeled goat anti-rabbit and rabbit anti-mouse secondary antibodies were from DAKO (Carpinteria, CA, USA). Chemiluminescence was detected using the ECL, SuperSignal West Pico, SuperSignal West Femto (Pierce) or the Lumi-Light Plus Western blotting kit (Roche) according to the manufacturer's instructions. Western blot analyses were performed at least three times.

Electron microscopy. Cells $\left(6 \times 10^{6}\right.$ per treatment group) were harvested and centrifuged at 1400 r.p.m. for $10 \mathrm{~min}$. Cell samples were then pre-fixed with $2.5 \%$ glutaraldehyde in $0.2 \mathrm{M}$ cacodylate buffer, $\mathrm{pH} 7.2$ for $20 \mathrm{~min}$ at room temperature. Following three washes with $0.2 \mathrm{M}$ cacodylate buffer, post-fixation of the samples was performed with $1 \%$ osmium tetroxide in $0.2 \mathrm{M}$ cacodylate buffer $\mathrm{pH} 7.2$ for $1 \mathrm{~h}$ at room temperature, and the cells washed again in $0.2 \mathrm{M}$ cacodylate buffer. Cells were then dehydrated through a graded series of ethanol solutions and embedded in Agar 100 (Agar Scientific, Essex, UK). Ultrathin sections obtained using a MT-2B ultramicrotome (LKB, Pharmacia, Uppsala, Sweden) were stained with uranyl acetate-lead citrate and examined with a Philips 208S electron microscope (FEI Corporation, Eindhoven, The Netherlands).

Xenograft tumor model. Inoculation of the human colon cancer HCT116 xenograft tumors was performed as described previously. ${ }^{37}$ Briefly, HCT116 cells $\left(1 \times 10^{6}\right)$ were subcutaneously injected into the right flank of $7-8$ weeks old female nu/nu mice (Charles River Laboratories, Wilmington, MA, USA). After 1 week, mice were randomized in four groups of eight mice and the indicated peptides were injected every day for 18 days by intraperitoneal injection. Tumor volume was measured using calipers in two dimensions. Primary tumor growth was calculated using the formula (width ${ }^{2} \times$ length) $/ 2$. At day 18 , mice were killed and tumors were taken for immunohistological study. Tissues were preserved in $10 \%$ formalin immediately after collection and processed into paraffin-embedded samples. Evaluation of apoptosis by TUNEL staining on slides was performed at the Rodent Histopathology Core Laboratory of the Dana-Farber/Harvard Cancer Center (Boston, MA, USA). Morphological analysis of liver samples was carried out using H\&E staining.

Trypan blue exclusion assay. Cells were cultured in 96-well plate. After treatment, cells in suspension were collected and mixed with $0.4 \%$ Trypan blue solution (Sigma-Aldrich) at a 1:1 ratio. In all, $10 \mu$ lof the cell suspension was then loaded onto TC10 System (Bio-Rad) counting slides and the number of viable cells quantified on a TC10 automated cell counter (Bio-Rad).

Cell viability assay. Cells were seeded in half-surface 96-well plates, treated as indicated and ATP concentrations were quantified using CellTiter-Glo reagent (Promega, Madison, WI, USA) according to the manufacturer's instructions. Luminescence was recorded using a Victor $3 \mathrm{~V}$ plate reader (Perkin Elmer, Boston, MA, USA).

Annexin V/7-AAD staining. Cells were seeded in 12-well plate and treated for $18 \mathrm{~h}$ before collection. Cells were then stained using the Annexin V-BrdU/FITC flow kit (BD Biosciences-Pharmingen, San Diego, CA, USA), according to the manufacturer's instructions. Stained cells were analyzed on a LSRII flow cytometer and FACSDiva software (BD Biosciences).

Particle radius of killerFLIP-E by DLS. Particle radius of killerFLIP micelles were determined using dynamic light scattering (DLS) measurements on a Dynapro Nanostar Wyatt laser photometer (Wyatt technology Corporation, Santa Barbara, CA, USA). In all, $60 \mu \mathrm{M}$ killerFLIP-E dissolved in PBS, pH 7.4 at $25^{\circ} \mathrm{C}$, was exposed to $90^{\circ}$ light scattering for $3 \mathrm{~min}$. All samples were filtered, degassed and scanned using a 1 -mm path length quartz cuvette. DLS data were analyzed by using the CONTIN method supplied by the manufacturer. 
Statistical analysis. All experiments were performed independently at least three times, except otherwise indicated. Statistical analyses were performed using two-tailed Student's $t$-tests. $P$-values $<0.05$ were considered significant and indicated with an asterisk.

\section{Conflict of Interest}

The authors declare no conflict of interest.

Acknowledgements. This work was supported by the National Institutes of Health grants CA105306, CA131664 and HL080192 to RK-F and GM054082 to DM. Octavian Bucur received a fellowship from the Lady TATA Memorial Trust, London, UK. This work was partially supported by Fondazione Peretti, Arcobaleno, AIRC MCO 9998 and 11505.

1. Alderton GK, Bordon Y. Tumour immunotherapy-leukocytes take up the fight. Nat Rev Immunol 2012; 12: 237.

2. Pecot CV, Calin GA, Coleman RL, Lopez-Berestein G, Sood AK. RNA interference in the clinic: challenges and future directions. Nat Rev Cancer 2011; 11: 59-67.

3. Vander Heiden MG. Targeting cancer metabolism: a therapeutic window opens. Nat Rev Drug Discov 2011; 10: 671-684.

4. Tu Z, Hao J, Kharidia R, Meng XG, Liang JF. Improved stability and selectivity of lytic peptides through self-assembly. Biochem Biophys Res Commun 2007; 361: 712-717.

5. Papo N, Shai Y. New lytic peptides based on the D,L-amphipathic helix motif preferentially kill tumor cells compared to normal cells. Biochemistry 2003; 42: 9346-9354.

6. Zhong J, Chau Y. Antitumor activity of a membrane lytic peptide cyclized with a linker sensitive to membrane type 1-matrix metalloproteinase. Mol Cancer Ther 2008; 7: 2933-2940.

7. Stewart ML, Fire E, Keating AE, Walensky LD. The MCL-1 BH3 helix is an exclusive MCL-1 inhibitor and apoptosis sensitizer. Nat Chem Biol 2010; 6: 595-601.

8. Rangel R, Guzman-Rojas L, le Roux LG, Staquicini FI, Hosoya H, Barbu EM et al. Combinatorial targeting and discovery of ligand-receptors in organelles of mammalian cells. Nat Commun 2012; 3: 788.

9. Dartois V, Sanchez-Quesada J, Cabezas E, Chi E, Dubbelde C, Dunn C et al. Systemic antibacterial activity of novel synthetic cyclic peptides. Antimicrob Agents Chemother 2005; 49: 3302-3310.

10. Lockshin RA, Zakeri Z. Cell death in health and disease. J Cell Mol Med 2007; 11: 1214-1224.

11. Jin TG, Kurakin A, Benhaga N, Abe K, Mohseni M, Sandra F et al. Fas-associated protein with death domain (FADD)-independent recruitment of c-FLIPL to death receptor 5. J Biol Chem 2004; 279: 55594-55601.

12. Ozturk S, Schleich K, Lavrik IN. Cellular FLICE-like inhibitory proteins (c-FLIPs): fine-tuners of life and death decisions. Exp Cell Res 2012; 318: 1324-1331.

13. Brooks H, Lebleu B, Vives E. Tat peptide-mediated cellular delivery: back to basics. Adv Drug Delivery Rev 2005; 57: 559-577.

14. Bechinger B, Lohner K. Detergent-like actions of linear amphipathic cationic antimicrobial peptides. Biochimica et biophysica acta 2006; 1758: 1529-1539.

15. Hoskin DW, Ramamoorthy A. Studies on anticancer activities of antimicrobial peptides. Biochimica et biophysica acta 2008; 1778: 357-375.

16. Teixeira V, Feio MJ, Bastos M. Role of lipids in the interaction of antimicrobial peptides with membranes. Prog Lipid Res 2012; 51: 149-177.

17. Pennarun B, Meijer A, de Vries EG, Kleibeuker JH, Kruyt F, de Jong S. Playing the DISC: turning on TRAIL death receptor-mediated apoptosis in cancer. Biochimica et biophysica acta 2010; 1805: 123-140.
18. Day TW, Huang S, Safa AR. c-FLIP knockdown induces ligand-independent DR5-, FADDcaspase-8-, and caspase-9-dependent apoptosis in breast cancer cells. Biochem Pharmacol 2008; 76: 1694-1704.

19. Peter ME. Programmed cell death: apoptosis meets necrosis. Nature $2011 ; 471$ : 310-312.

20. Matsuzaki K. Why and how are peptide-lipid interactions utilized for self-defense? Magainins and tachyplesins as archetypes. Biochimica et biophysica acta 1999; 1462 : $1-10$.

21. Shai $Y$. Mechanism of the binding, insertion and destabilization of phospholipid bilayer membranes by alpha-helical antimicrobial and cell non-selective membrane-lytic peptides. Biochimica et biophysica acta 1999; 1462: 55-70.

22. Sasisekharan R, Raman R, Prabhakar V. Glycomics approach to structure-function relationships of glycosaminoglycans. Annu Rev Biomed Engineering 2006; 8: 181-231.

23. Rabenstein DL. Heparin and heparan sulfate: structure and function. Natural Product Rep 2002; 19: 312-331.

24. Kleeff J, Ishiwata T, Kumbasar A, Friess H, Buchler MW, Lander AD et al. The cell-surface heparan sulfate proteoglycan glypican-1 regulates growth factor action in pancreatic carcinoma cells and is overexpressed in human pancreatic cancer. J Clin Investig 1998; 102: $1662-1673$.

25. Matsuda K, Maruyama H, Guo F, Kleeff J, Itakura J, Matsumoto $Y$ et al. Glypican-1 is overexpressed in human breast cancer and modulates the mitogenic effects of multiple heparin-binding growth factors in breast cancer cells. Cancer Res 2001; 61: 5562-5569.

26. Nakatsura T, Kageshita T, Ito S, Wakamatsu K, Monji M, Ikuta $Y$ et al. Identification of glypican-3 as a novel tumor marker for melanoma. Clin Cancer Res 2004; 10: 6612-6621.

27. Fadnes $B$, Rekdal $O$, Uhlin-Hansen L. The anticancer activity of lytic peptides is inhibited by heparan sulfate on the surface of the tumor cells. BMC Cancer 2009; 9: 183.

28. Fadnes B, Uhlin-Hansen L, Lindin I, Rekdal O. Small lytic peptides escape the inhibitory effect of heparan sulfate on the surface of cancer cells. BMC Cancer 2011; 11: 116.

29. Urbinati C, Nicoli S, Giacca M, David G, Fiorentini S, Caruso A et al. HIV-1 Tat and heparan sulfate proteoglycan interaction: a novel mechanism of lymphocyte adhesion and migration across the endothelium. Blood 2009; 114: 3335-3342.

30. Hsu CC, Lin TW, Chang WW, Wu CY, Lo WH, Wang PH et al. Soyasaponin-I-modified invasive behavior of cancer by changing cell surface sialic acids. Gynecol Oncol 2005; 96: 415-422.

31. Ulloa F, Real FX. Differential distribution of sialic acid in alpha2,3 and alpha2,6 linkages in the apical membrane of cultured epithelial cells and tissues. J Histochem Cytochem 2001; 49: 501-510.

32. Schwartz MA. Integrins, oncogenes, and anchorage independence. J Cell Biol 1997; 139: $575-578$.

33. Dusek RL, Attardi LD. Desmosomes: new perpetrators in tumour suppression. Nat Rev Cancer 2011; 11: 317-323.

34. Del Gaizo V, MacKenzie JA, Payne RM. Targeting proteins to mitochondria using TAT Mol Genet Metabolism 2003; 80: 170-180.

35. Tait SW, Green DR. Mitochondria and cell death: outer membrane permeabilization and beyond. Nat Rev Mol Cell Biol 2010; 11: 621-632.

36. Fjell CD, Hiss JA, Hancock RE, Schneider G. Designing antimicrobial peptides: form follows function. Nat Rev Drug Discov 2012; 11: 37-51.

37. Song K, Benhaga N, Anderson RL, Khosravi-Far R. Transduction of tumor necrosis factorrelated apoptosis-inducing ligand into hematopoietic cells leads to inhibition of syngeneic tumor growth in vivo. Cancer Res 2006; 66: 6304-6311.

(1) (\$) Cell Death and Disease is an open-access journal published by Nature Publishing Group. This work is licensed under a Creative Commons Attribution-NonCommercialNoDerivs 3.0 Unported License. To view a copy of this license, visit http://creativecommons.org/licenses/by-nc-nd/3.0/

\section{Supplementary Information accompanies this paper on Cell Death and Disease website (http://www.nature.com/cddis)}

